\title{
Effects of empagliflozin on cardiorespiratory fitness and significant interaction of loop diuretics
}

\author{
Salvatore Carbone $\mathrm{PhD}^{1,2} \odot$ | Justin M. Canada MS ${ }^{1,3}$ | Hayley E. Billingsley RD ${ }^{1}$ | \\ Dinesh Kadariya MD ${ }^{1}$ | Dave L. Dixon PharmD ${ }^{4}$ | Cory R. Trankle MD ${ }^{1}$ | \\ Leo F. Buckley PharmD ${ }^{5}$ | Roshanak Markley MD ${ }^{1}$ | Chau Vo MD ${ }^{1}$ | \\ Horacio Medina de Chazal MD ${ }^{1}$ | Sanah Christopher MD $^{1}$ | Raffaella Buzzetti MD ${ }^{2}$ | \\ Benjamin W. Van Tassell PharmD ${ }^{4}$ | Antonio Abbate MD $^{1}$
}

\author{
${ }^{1}$ Department of Internal Medicine, VCU \\ Pauley Heart Center, Virginia Commonwealth \\ University, Richmond, Virginia \\ ${ }^{2}$ Department of Experimental Medicine, \\ Sapienza University of Rome, Rome, Italy \\ ${ }^{3}$ Kinesiology and Health Science, College of \\ Humanities and Sciences, Virginia \\ Commonwealth University, Richmond, Virginia \\ ${ }^{4}$ Department of Pharmacotherapy and \\ Outcome Sciences, Virginia Commonwealth \\ University, Richmond, Virginia \\ ${ }^{5}$ Division of Cardiovascular Medicine and \\ Department of Pharmacy Service, Brigham and \\ Women's Hospital, Boston, Massachusetts

\section{Correspondence} \\ Salvatore Carbone PhD, Research Instructor of \\ Medicine, VCU Pauley Heart Center, Virginia \\ Commonwealth University, West Hospital, 5th \\ Floor, Room 520, 1200 E Broad Street, \\ P.O. Box 980204 , Richmond, Virginia 23298. \\ Email: salvatore.carbone@vcuhealth.org \\ Funding information \\ This project was supported by a Mentored \\ Clinical and Population Research Award \\ (16MCPRP31100003) from the American \\ Heart Association (S. C.) and, in part, by a CTSA \\ award (UL1TR000058) from the National \\ Center for Advancing Translational Sciences.
}

The effects of empagliflozin on cardiorespiratory fitness in patients with type 2 diabetes mellitus (T2DM) and heart failure with reduced ejection fraction (HFrEF) are unknown. In this pilot study we determined the effects of empagliflozin $10 \mathrm{mg} / \mathrm{d}$ for 4 weeks on peak oxygen consumption $\left(\mathrm{VO}_{2}\right)$ in 15 patients with T2DM and HFrEF. As an exploratory analysis, we assessed whether there was an interaction of the effects of empagliflozin on peak $\mathrm{VO}_{2}$ of loop diuretics. Empagliflozin reduced body weight $(-1.7 \mathrm{~kg} ; P=.031)$, but did not change peak $\mathrm{VO}_{2}$ (from $14.5 \mathrm{~mL} \mathrm{~kg}^{-1} \mathrm{~min}^{-1}$ [12.6-17.8] to 15.8 [12.5-17.4] $\mathrm{mL} \mathrm{kg}^{-1} \mathrm{~min}^{-1} ; P=.95$ ). However, patients using loop diuretics $(\mathrm{N}=9)$ demonstrated an improvement, whereas those without loop diuretics $(\mathrm{N}=6)$ experienced a decrease in peak $\mathrm{VO}_{2}(+0.9$ [0.1-1.4] vs -0.9 [ -2.1 to -0.3$] \mathrm{mL}$ $\mathrm{kg}^{-1} \mathrm{~min}^{-1} ; P=.001$ ), and peak $\mathrm{VO}_{2}$ changes correlated with the baseline daily dose of diuretics $(R=+0.83 ; P<.001)$. Empagliflozin did not improve peak $V_{2}$ in patients with T2DM and HFrEF. However, as a result of exploratory analysis, patients concomitantly treated with loop diuretics experienced a significant improvement in peak $\mathrm{VO}_{2}$.

\section{KEYWORDS}

cardiorespiratory fitness, diuretics, empagliflozin, heart failure, SGLT2 inhibitors

\section{1 | INTRODUCTION}

Sodium glucose co-transporter (SGLT)-2 inhibitors such as empagliflozin and canagliflozin reduced the incidence of heart failure (HF) and HF hospitalizations in clinical trials including patients with type 2 diabetes mellitus (T2DM). ${ }^{1,2} \mathrm{HF}$, like T2DM, is a chronic debilitating condition, and is associated with impaired functional capacity and reduced quality of life. The effects of SGLT-2 inhibitors on cardiorespiratory fitness (CRF) in patients with HF with reduced ejection fraction (HFrEF) are unknown. ${ }^{3}$
In this pilot study (ClinicalTrials.gov Identifier: NCT02862067), we hypothesized that empagliflozin would improve CRF, measured as changes in peak oxygen consumption $\left(\mathrm{VO}_{2}\right)$ at maximal cardiopulmonary exercise test (CPX) in patients with HFrEF and T2DM. Patients with T2DM and HFrEF share an exaggerated neurohormonal system activation, in particular, of the renin-angiotensin-aldosterone system (RAAS), which is a major driver of CRF and clinical outcomes in HFrEF, independent of the blood pressure-lowering effects. ${ }^{4}$ By reducing glucose reabsorption in the proximal renal tubules and increasing the concentration of sodium at macula densa level, SGLT-2 inhibitors may 
prevent renin secretion and overall RAAS activation in response to reduced sodium tubular levels. We therefore hypothesized that, despite increased diuresis and natriuresis, empagliflozin would not further activate the renin-angiotensin-aldosterone system (RAAS), known to be involved in the pathophysiology of HFrEF.

Finally, as an exploratory analysis, we planned to determine whether the effects of empagliflozin were influenced by concomitant treatment with loop diuretics, agents also known to promote natriuresis and commonly used in HFrEF to manage symptoms.

\section{2 | METHODS}

\subsection{Cardiorespiratory fitness and physical activity assessments}

In this open-label single-arm prospective study, we assessed the effects of empagliflozin on peak $\mathrm{VO}_{2}$ with $\mathrm{CPX}$ in 15 patients with stable symptomatic HFrEF (New York Heart Association [NYHA] class II-III, left ventricular EF $[\mathrm{LVEF}]<50 \%$ ) and T2DM (glycosylated haemoglobin [HbA1c] 6.5\%-10.0\%) using a metabolic cart interfaced with a treadmill and a conservative ramping protocol before and after treatment with empagliflozin $10 \mathrm{mg}$ daily for 4 weeks, in which speed and grade were increased by approximately 0.6 estimated metabolic equivalents (MET) every 60 seconds. ${ }^{5,6}$ Ventilatory gas analysis was performed using a VMax Encore metabolic cart (Carefusion, Yorba Linda, California) with a standard mouthpiece and nose clip setup. Calibration of the metabolic cart for volume and gas concentration was obtained before every test. Ventilatory gas analysis measurements were obtained for at least 3 minutes in the seated position before beginning exercise, continuously throughout exercise, and 2 minutes into the recovery period. Blood pressure (BP) was monitored with a Tango exercise BP system (Suntech Medical, Morrisville, North Carolina). BP measurements were monitored at rest, during exercise, and during the recovery period. Resting BP was measured according to clinical recommendations and using standardized methods. In brief, patients rested in a seated position for at least 10 minutes; an appropriately sized brachial artery pressure cuff was used, and pressure was measured with an automated device (Tango system, Suntech Medical, Morrisville, North Carolina).

Peak $\mathrm{VO}_{2}$ is a strong independent predictor of events and is sensitive for detecting the efficacy of therapeutic interventions in HF patients. ${ }^{5}$ Importantly, peak $\mathrm{VO}_{2}$, measured by ventilatory expired gas analysis, is not biased by overexertion, and is insensitive to the placebo effect. $^{5,7}$

Physical activity was assessed using the International Physical Activity Questionnaire-short version (IPAQ). The IPAQ-short is a 7-question questionnaire that estimates physical activity by assessing responses to questions concerning duration and intensity of daily physical activity.

\section{2 | Cardiac function assessment}

We assessed cardiac function using transthoracic Doppler echocardiography to measure LV end-diastolic and end-systolic volume, LVEF, early transmitral velocities (E) at pulsed wave Doppler spectra, early mitral annulus velocities by tissue Doppler averaged between lateral and septal $\left(\mathrm{e}^{\prime}\right)$, and we calculated the $\mathrm{E} / \mathrm{e}^{\prime}$ ratio. ${ }^{8,9}$

\section{3 | Diuresis, natriuresis and RAAS activation assessments}

We assessed the effects of empagliflozin on 24-hour diuresis, urinary sodium concentration ( $\mathrm{Na}^{+} \mathrm{UR}$ ) and RAAS activation, measured as plasma renin activity (PRA), aldosterone plasma levels (ALD) and ALD/PRA ratio, following overnight fasting and after suspension of angiotensin II antagonists (ATII-A) and mineralocorticoid-receptor antagonists (MRA) for 24 hours. Chemical laboratory analyses were performed using clinical standard assays (LabCorp, Burlington, North Carolina).

\subsection{Dietary sodium, body weight and waist circumference assessments}

Dietary sodium intake can affect fluid status and RAAS activation; therefore, we estimated sodium intake using a standardized 5-pass interview 24-hour dietary recall, as previously described. ${ }^{10}$ Body weight and waist circumference were measured on the day of RAAS activation assessment. Waist circumference was measured according to the World Health Organization recommendations. ${ }^{11}$

\section{5 | Statistical analysis}

We used SPSS 24.0 (IBM Corp) for statistical analysis. Data are reported as median and interquartile range for potential deviation from the Gaussian distribution. Discrete variables are reported as a number and percentage. Interval changes between baseline and 4 weeks in peak $\mathrm{VO}_{2}$ were analysed using the Wilcoxon test. Analysis of interaction was assessed with a general linear model for repeated measures for discrete variables or the Spearman correlation rank test for continuous variables. Multivariate analysis using a linear regression model was performed, using a stepwise approach including those variables associated with $P<.05$ at univariate analysis.

The Virginia Commonwealth University Institutional Review Board approved the study, and all patients provided written informed consent.

\section{3 | RESULTS}

\section{1 | Baseline characteristics}

Among the patients, 8 (53\%) were women, 9 (60\%) were White and 6 (40\%) were Black Americans, median age was 60 (56-62) years, and body mass index (BMI) was $34(31-37) \mathrm{kg} / \mathrm{m}^{2}$. All patients were using ATII-A; 7 patients (47\%) were using MRA and 9 (60\%) patients were using loop diuretics. Median HbA1c was 7.8\% (7.2-8.6), NTproBNP was $229 \mathrm{pg} / \mathrm{mL}$ (47-657), creatinine was $1.1 \mathrm{mg} / \mathrm{dL}$ (0.9-1.2), estimated glomerular filtration rate was $63 \mathrm{~mL} / \mathrm{min} / 1.73 \mathrm{~m}^{2}$ (63-88), PRA was $5.4 \mathrm{ng} / \mathrm{mL} / \mathrm{h}$ (2.5-20.0) and ALD was $9.3 \mathrm{ng} / \mathrm{dL}$ (6.8-13.7). 
Median peak $\mathrm{VO}_{2}$ was $14.5 \mathrm{~mL} \mathrm{~kg}^{-1} \mathrm{~min}^{-1}$ (12.6-17.8) or $57 \%$ (48-60) of predicted normative values. ${ }^{5}$ Median LVEF was $41 \%$ (33-43), E/e $\mathrm{e}^{\prime}$ was 10.9 (10.6-19.5) and $\mathrm{e}^{\prime}$ was $6.5 \mathrm{~cm} / \mathrm{s}$ (5.5-7.4). Median 24-hour urinary volume excretion was $1725 \mathrm{~mL}$ (1307-2354) and median $\mathrm{Na}^{+} \mathrm{UR}$ was $72.5 \mathrm{mmol} / \mathrm{L}$ (64.9-110.8). Median dietary sodium intake was $2869 \mathrm{mg}$ (1701-3846).

\section{2 | Body weight, fasting glycaemia, blood pressure and cardiorespiratory fitness}

In the overall cohort, treatment with empagliflozin for 4 weeks increased 24-hour urine output $(+43 \% ; P=.007)$, reduced body weight $(-1.7 \mathrm{~kg}, P=.031)$, reduced resting diastolic blood pressure $(-8 \mathrm{~mm} \mathrm{Hg} ; P=.04)$ and led to a small, although statistically significant, reduction in E/e' ratio (from 10.9 [10.6-19.5] to 9.8 [8.3-16.9]; $P=.035$ ).

Empagliflozin did not, however, change peak $\mathrm{VO}_{2}$ relative to body weight (from 14.5 [12.6-17.8] to 15.8 [12.5-17.4] $\mathrm{mL} \mathrm{kg}^{-1} \mathrm{~min}^{-1}$; $P=.95$ ) or when expressed in absolute values (from 1426 [1037-1672] to 1517 [1104-1726] $\mathrm{mL} \mathrm{min}^{-1} ; P=.95$ ) in the overall cohort, nor did it change IPAQ-estimated physical activity $(P=.59)$. We found a large variability in changes in peak $\mathrm{VO}_{2}$, ranging from -2.9 to $+2.5 \mathrm{~mL} \mathrm{~kg}^{-1} \mathrm{~min}^{-1}$. None of the clinical variables, echocardiographic LV parameters or changes in IPAQ-estimated physical activity correlated with changes in peak $\mathrm{VO}_{2}$ with empagliflozin (all $P>.05$ ). Moreover, neither the doses of ATII-A or MRA, nor the use of MRA, influenced the changes in peak $\mathrm{VO}_{2}$ with empagliflozin. Importantly, dietary sodium intake did not change during the overall duration of the study (from 2869 [1701-3846] to 2571 [2098-3378] mg; $P=.39$ ). Treatment with empagliflozin for 4 weeks did not affect fasting glycaemia (from 140 [129-220] to 144 [107-166] mg/dL; $P=.16$ ).

\subsection{Loop diuretic use and effects on peak $\mathrm{VO}_{2}$}

A statistically significant interaction between loop diuretic use (9 [60\%]) and effects on peak $\mathrm{VO}_{2}$ was found, for both peak $\mathrm{VO}_{2}$ relative to body weight (Figure $1 \mathrm{~A})(P=.001)$ and absolute peak $\mathrm{VO}_{2}$ $(P=.001)$, showing a dose-dependent relationship between diuretic dose and peak $\mathrm{VO}_{2}$ changes $(\mathrm{R}=+0.83 ; P<.001)$ (Figure 1B), without differences in physical activity and blood pressure changes between the 2 groups $\left(P=.28\right.$ and $P=.77$, respectively). The change in $\mathrm{Na}^{+} \mathrm{UR}$ (Figure $1 C$ ) and in the ALD/PRA ratio also correlated positively with changes in peak $\mathrm{VO}_{2}(\mathrm{R}=+0.622 ; P=.023$ and $\mathrm{R}=+0.52 ; P=0.057$, respectively). Neither ATII-A nor MRA doses correlated with changes in $\mathrm{Na}^{+} \mathrm{UR}, \mathrm{PRA}$ or ALD/PRA (all $P>$.05).

When investigating potential contributors to peak $\mathrm{VO}_{2}$ changes in the overall cohort, the statistically significant predictors at univariate analysis were $\mathrm{BMI}$, waist circumference, $\mathrm{HbA} 1 \mathrm{c}$ and loop diuretic dose, but diuretic dose remained the only significant predictor for peak $\mathrm{VO}_{2}$ changes at multivariate analysis ( $\beta$ coefficient $=.623$; $P=.013)$.

A significant interaction between diuretic use and the effects of empagliflozin on $\mathrm{Na}^{+} U \mathrm{R}$ was also found, showing a reduction in $\mathrm{Na}^{+} U R$, a surrogate for renin activation ${ }^{12}$ in patients treated with empagliflozin without loop diuretics, but not in those treated with loop diuretics (-32\% vs $0 \%$, respectively; $P=.031$ ). We found a non-
(A)

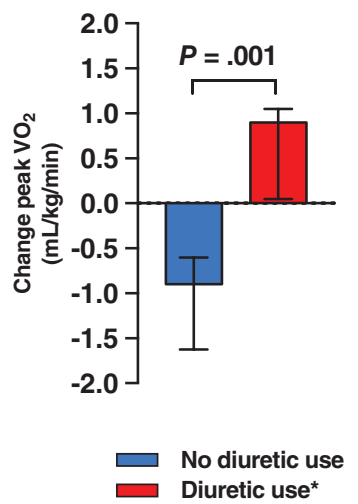

(B)

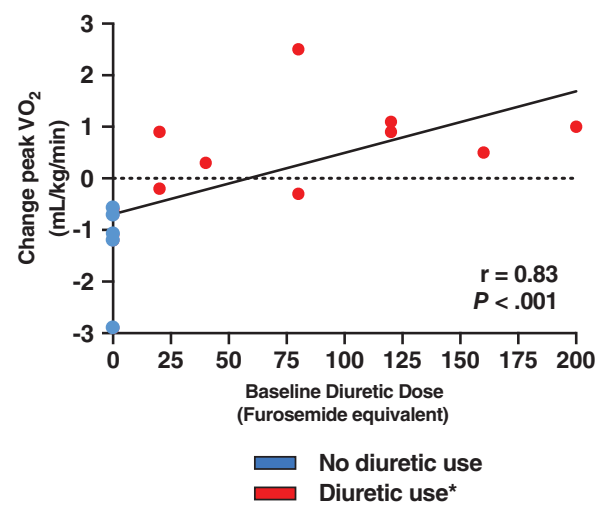

FIGURE 1 Greater peak oxygen consumption $\left(\mathrm{VO}_{2}\right)$ changes in patients with heart failure and reduced ejection fraction (HFrEF) receiving loop diuretics (A). Baseline diuretic dose $(B)$ and changes in urinary sodium concentration ( $\left.\mathrm{Na}^{+} \mathrm{UR}\right)$ (C) correlate with change in peak $\mathrm{VO}_{2}$. Increased aldosterone (ALD)/renin ratio in patients receiving loop diuretics (D). Panel $C$ excludes 2 patients not treated with diuretic because of laboratory internal issues for 24-hour urinary sodium concentration measurement, possibly the result of damaged samples. *diuretic use limited to loop diuretic use and expressed as furosemide-equivalent dose $(\mathrm{mg} / \mathrm{d})$

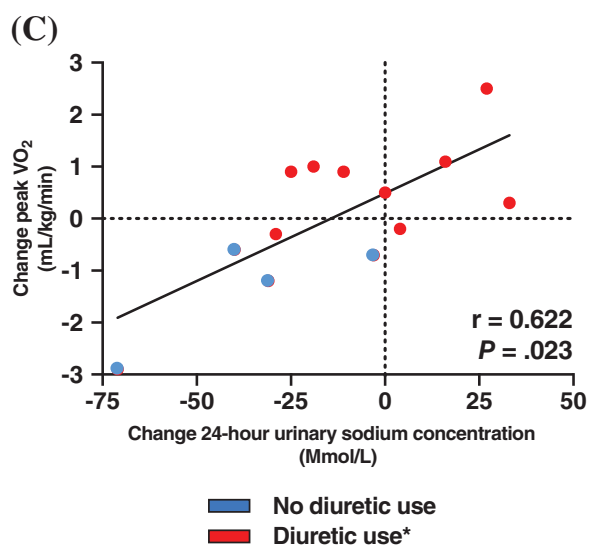

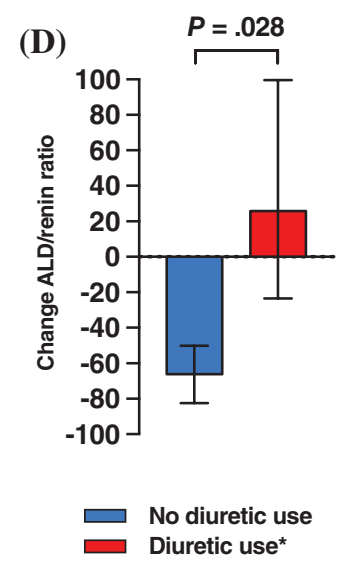


significant trend for PRA changes without and with diuretics (+50\% vs $-22 \%$, respectively; $P=.21$ ) and a significant interaction between use of diuretics and the ALD/PRA ratio, a more accurate marker for renin activation (decrease in ratio) vs aldosterone hypersecretion (increase) ( $-66 \%$ vs $+26 \%$, respectively; $P=.028$ ) (Figure $1 D)$. Finally, we found a significant association between changes in resting diastolic BP and aldosterone plasma level changes $(R=-0.55 ; P=.03)$. However, neither systolic nor diastolic resting BP were associated with changes in CRF (both $P>.30$ ).

\section{4 | DISCUSSION}

The data presented herein show, for the first time, that SGLT-2 inhibition with empagliflozin was associated with a highly heterogeneous response in terms of peak $\mathrm{VO}_{2}, \mathrm{Na}^{+} U R$, PRA and ALD/PRA that depended on the presence or absence of concomitant loop diuretic use. Although resulting from an exploratory analysis requiring confirmation in larger clinical trials, the combination of empagliflozin and loop diuretics appears to have synergistic effects on diuresis, without inducing RAAS activation, and resulting in an increase in $\mathrm{Na}^{+} U \mathrm{R}$ concentration, and in a significant increase in peak $\mathrm{VO}_{2}$. On the other hand, the use of empagliflozin without loop diuretics induced a paradoxical reduction in $\mathrm{Na}^{+} U R$, resulting in more diluted urine, possibly as the result of RAAS activation, and in a lack of any improvement, or even a reduction, in peak $\mathrm{VO}_{2}$. It is therefore possible that empagliflozin improves $\mathrm{CRF}$ in patients concomitantly treated with loop diuretics by reducing RAAS activation, which is well-known to be detrimental in HFrEF, but also in T2DM. Inhibition of RAAS activation reduces systemic vascular resistance, resulting in vasodilation, improved cardiac output, improved ventilatory efficiency, increased natriuresis/ diuresis and improved CRF.,13

Our study is however, limited by the small sample size and by the absence of assessment of $\mathrm{O}_{2}$ delivery and/or utilization, which could have also affected the overall results, including the absence of significant improvements in fasting glycaemia. Specifically, that may be the result of our study being underpowered to detect changes in fasting glycaemia. However, we cannot exclude the possibility that the effects of empagliflozin in patients with established HFrEF are less pronounced than in non-HFrEF patients, or that it requires more time to achieve a similar effect, clearly requiring further and larger studies, specifically in the HFrEF population. ${ }^{14}$ Moreover, changes in CRF did not simply reflect interval changes in physical activity which, however, was estimated using a subjective questionnaire that may not be as sensitive as other means such as accelerometers.

SGLT-2 inhibitors reduced HF-related events in randomized clinical trials ${ }^{1,2}$ and in real-world data ${ }^{15}$; however, the effects of this class in well-characterized patients with established HFrEF, and more specifically, the effects on peak $\mathrm{VO}_{2}$, remain unknown.

Here we report an interaction between empagliflozin and use of loop diuretics in patients with HFrEF, an interaction that was interestingly posited in a recent clinical trial. ${ }^{2,16}$ The implications would be that the use of empagliflozin in HFrEF patients not treated with loop diuretics may be less beneficial. If confirmed in larger studies evaluating the role of SGLT-2 inhibitors, specifically in patients with $\mathrm{HFrEF}$, this could greatly influence the clinical applications of these agents.

\section{ACKNOWLEDGMENT}

We would like to thank Peter Westman, Andy R. Nguyen, Yasaman Ataeijannati, Marco Del Buono, Michele Viscusi, Nicola Potere and Veronica Adiletta for their technical support and their help with data collation.

\section{Conflict of interest}

A. A. has received research grants from and has served on an advisory board for Janssen. The other authors have nothing to disclose.

\section{Author contributions}

Study Design: Salvatore Carbone \& Antonio Abbate. Study conduction: Salvatore Carbone, Justin M Canada, Hayley E Billingsley, Dinesh Kadariya, Dave L Dixon, Cory R Trankle, Leo F Buckley, Roshanak Markley, Chau Vo, Horacio Medina de Chazal, Sanah Christopher \& Antonio Abbate. Data analysis: Salvatore Carbone \& Antonio Abbate. Manuscript writing: Salvatore Carbone, Justin M Canada, Hayley E Billingsley, Dave L Dixon, Cory R Trankle, Leo F Buckley, Raffaella Buzzetti, Benjamin W Van Tassell \& Antonio Abbate.

\section{ORCID}

Salvatore Carbone (iD) http://orcid.org/0000-0002-8163-0527

Leo F. Buckley (D) http://orcid.org/0000-0003-1570-1486

\section{REFERENCES}

1. Zinman B, Wanner C, Lachin JM, et al. Empagliflozin, cardiovascular outcomes, and mortality in type 2 diabetes. N Engl J Med. 2015;373: 2117-2128.

2. Neal B, Perkovic V, Mahaffey KW, et al. Canagliflozin and cardiovascular and renal events in type 2 diabetes. N Engl J Med. 2017;377:644-657.

3. Butler J, Anker SD. The ethics of conducting clinical trials with sodium-glucose cotransporter-2 inhibitors in heart failure. Circulation. 2017;136:1459-1461.

4. Abbate A, Van Tassell BW, Canada JM, Dixon DL, Arena RA, Biondi-Zoccai G. Pharmacologic and surgical interventions to improve functional capacity in heart failure. Heart Fail Clin. 2015;11:117-124.

5. Guazzi M, Bandera F, Ozemek C, Systrom D, Arena R. Cardiopulmonary exercise testing. J Am Coll Cardiol. 2017;70:1618-1636.

6. Canada JM, Fronk DT, Cei LF, et al. Usefulness of C-reactive protein plasma levels to predict exercise intolerance in patients with chronic systolic heart failure. Am J Cardiol. 2015;117:2-6.

7. Van Tassell BW, Arena RA, Toldo S, et al. Enhanced interleukin-1 activity contributes to exercise intolerance in patients with systolic heart failure. PLoS One. 2012;7:e33438.

8. Lang RM, Badano LP, Mor-Avi V, et al. Recommendations for cardiac chamber quantification by echocardiography in adults: an update from the American Society of Echocardiography and the European Association of Cardiovascular Imaging. Eur Heart J - Cardiovasc Imaging. 2015; 16:233-271.

9. Nagueh SF, Smiseth OA, Appleton CP, et al. Recommendations for the evaluation of left ventricular diastolic function by echocardiography: an update from the American Society of Echocardiography and the European Association of Cardiovascular Imaging. Eur Heart J - Cardiovasc Imaging. 2016;17:1321-1360. 
10. Carbone S, Canada JM, Buckley LF, et al. Dietary fat, sugar consumption and cardiorespiratory fitness in patients with heart failure with preserved ejection fraction. JACC Basic to Transl Sci. 2017;2:513-525.

11. World Health Organization. Waist Circumference and Waist-Hip Ratio: Report of a WHO Expert Consultation, Geneva: World Health Organization; 2011; 8-11 December 2008. http://www.who.int/nutrition/ publications/obesity/WHO_report_waistcircumference_and_waisthip _ratio/en/. Accessed March 11, 2018.

12. Marenzi G, Lauri G, Assanelli E, et al. Serum to urinary sodium concentration ratio is an estimate of plasma renin activity in congestive heart failure. Eur J Heart Fail. 2002;4:597-603.

13. Creager MA, Massie BM, Faxon DP, et al. Acute and long-term effects of enalapril on the cardiovascular response to exercise and exercise tolerance in patients with congestive heart failure. J Am Coll Cardiol. 1985;6:163-173.

14. Butler J, Hamo CE, Filippatos G, et al. The potential role and rationale for treatment of heart failure with sodium-glucose co-transporter 2 inhibitors. Eur J Heart Fail. 2017;19:1390-1400.
15. Kosiborod M, Cavender MA, Fu AZ, et al. Lower risk of heart failure and death in patients initiated on sodium-glucose Cotransporter-2 inhibitors versus other glucose-lowering drugs clinical perspective. Circulation. 2017;136:249-259.

16. Rådholm K, Figtree G, Perkovic V, et al. Canagliflozin and heart failure in type 2 diabetes mellitus: results from the CANVAS program (Canagliflozin Cardiovascular Assessment Study). Circulation. 2018. https://doi.org/10.1161/CIRCULATIONAHA.118.034222 [Epub ahead of print].

How to cite this article: Carbone S, Canada JM, Billingsley $\mathrm{HE}$, et al. Effects of empagliflozin on cardiorespiratory fitness and significant interaction of loop diuretics. Diabetes Obes Metab. 2018;1-5. https://doi.org/10.1111/dom.13309 